\title{
Hyperbolic Coxeter Pyramids
}

\author{
John Mcleod \\ Department of Mathematical Sciences, University of Durham, Durham, England \\ Email: j.a.mcleod@durham.ac.uk
}

Received August 11, 2012; revised October 10, 2012; accepted October 21, 2012

\begin{abstract}
Hyperbolic Coxeter polytopes are defined precisely by combinatorial type. Polytopes in hyperbolic $n$-space with $n+p$ faces that have the combinatorial type of a pyramid over a product of simplices were classified by Tumarkin for small $p$. In this article we generalise Tumarkin's methods and find the remaining hyperbolic Coxeter pyramids.
\end{abstract}

Keywords: Hyperbolic; Coxeter; Polytope; Pyramid

\section{Introduction}

The polytopes in $n$ dimensions that have $n+1$ faces are referred to collectively as the simplices of the geometric space they inhabit. A simplex may always be thought of as a pyramid, although every face may be considered to be the "base" of the pyramid. In this article we consider only Coxeter polytopes, which are precisely those that are the fundamental domains of reflection groups. The Coxeter simplices are well known in Euclidean, Spherical and Hyperbolic space. These lists illustrate an important distinction that separates Hyperbolic space from the first two spaces in this list, namely that there is an upper bound on the dimension above which there are no simplices.

This distinction is much stronger than the example illustrates. The proof due to Vinberg that there are no co-compact hyperbolic reflection groups for $n \geq 30$ is principally a combinatorial proof demonstrating that there are no hyperbolic Coxeter polytopes for large enough dimension (c.f. [1]).

The bounded hyperbolic Coxeter simplices were classified by Lannér [2] in 1950. The non-compact hyperbolic Coxeter simplices can be enumerated using similar methods to Lannér. These have been well studied (c.f. [3-6]).

Let $P$ be a Coxeter polytope in hyperbolic space $H^{n}$ with $n+p$ faces. For $p>1$ complete lists of hyperbolic Coxeter polytopes have been published by Tumarkin: $p=2$ in [7]; and $p=3$ in [8]. These lists are complete, and contain in their number many examples of hyperbolic Coxeter polytopes with the combinatorial type of a pyramid. Tumarkin's technique made use of the Gale diagram (c.f. [9]) which has quantitatively different characteristics when it describes a pyramid, as compared to other configurations. This distinction led to pyramids being classified using separate methods originally due to Vinberg.

In this article we generalise Tumarkin's classification of Hyperbolic Coxeter pyramids in terms of the Coxeter diagram, and then find all remaining examples of such polytopes using simple combinatorial arguments. The relevant background about Coxeter diagrams and the polytopes they represent is presented in Section 2. In Section 3 we generalise the appropriate results of Tumarkin, and complete the classification of hyperbolic Coxeter polytopes whose combinatorial type is a pyramid over a product of simplices.

\section{Coxeter Diagrams}

An acute-angled polytope is called a Coxeter polytope if all the dihedral angles at the intersections of pairs of faces are integer submultiples of $\pi$ (or zero). A complete presentation of an acute-angled polytope is given by a Gram matrix. Denote by $\Pi_{i}$ the codimension one hyperplane containing the ith face of the polytope. A Gram matrix $G=g_{i j}$ is a symmetric matrix with entries:

$$
g_{i j}= \begin{cases}1, & \text { if } i=j \\ -\cos (\pi / n), & \text { if } \angle\left(\Pi_{i}, \Pi_{j}\right)=\pi / n \\ -1, & \text { if } \angle\left(\Pi_{i}, \Pi_{j}\right)=0 \\ -\cosh \left(\rho\left(\Pi_{i}, \Pi_{j}\right)\right), & \text { if } \Pi_{i} \text { and } \Pi_{j} \text { do not intersect }\end{cases}
$$

where $\rho\left(\Pi_{i}, \Pi_{j}\right)$ is the minimum hyperbolic distance between the two hyperplanes which contain the two faces.

A Coxeter diagram is an edge-labelled graph which represents almost all of the same information about the combinatorial structure of a Coxeter polytope. Each ver- 
tex of a Coxeter diagram corresponds to a face, and the labels for the edges are as presented in Table 1.

An edge corresponding to a dihedral angle of $\pi / m$ is said to be of weight $m$. The number of vertices in a Coxeter diagram is called the order of the diagram. A connected Coxeter diagram is called elliptic (respectively parabolic) if the corresponding Gram matrix is positive definite (respectively semidefinite and degenerate). A Coxeter diagram that consists only of elliptic (respectively parabolic) connected components is called elliptic (respectively parabolic). The rank of a Coxeter diagram is equal to the rank of the Gram matrix. The Gram matrix of a disconnected Coxeter diagram can be transformed into a block diagonal matrix via permutations of the rows and the same permutations of the columns. Therefore the rank of a disconnected Coxeter diagram is the sum of the ranks of its connected components. The rank of a connected elliptic diagram is equal to its order, while the rank of a connected parabolic diagram is one less than its order. Complete lists of connected elliptic and parabolic Coxeter diagrams can be found in [10].

The vertices of the Coxeter polytope $P$ can be read from the Coxeter diagram. Let $J$ denote the set of vertices of the Coxeter diagram, and $S_{I}$ the subdiagram corresponding to a subset $I \subset J$. We say that such a subset determines a face of the Coxeter polytope if the intersection of the faces in $I$ is a face of $P$. We recall the following proposition (in this form) from [8], originally proven in [1] as Theorems 3.1 and 3.2.

Proposition 2.1. ([8], Proposition 1)

1) A subset $I \subset J$ determines a face of the polytope $P$ (apart from an infinitely distant vertex) if and only if the subdiagram $S_{I}$ is elliptic. In this case the codimension of the corresponding face is the number of elements in $I$;

2) A subset $I \subset J$ determines an infinitely distant vertex if and only if the subdiagram $S_{I}$ is not elliptic and there is a subset $I^{\prime}$ such that $I \subset I^{\prime} \subset J$ and $S_{I^{\prime}}$ is parabolic of rank $n-1$.

We can see from this proposition that if the order of a Coxeter diagram which determines a face of $P$ is greater than $n$ it must correspond to an infinitely distant vertex, conversely if the order of a diagram which determines a face is less than $n$ it must be elliptic.

Table 1. The edges of a Coxeter diagram.

\begin{tabular}{cc}
\hline Type of edge: & Corresponds to: \\
\hline $\begin{array}{c}\text { Comprised of } m-2 \text { lines, or } \\
\text { labeled } m\end{array}$ & A dihedral angle $\pi / m$ \\
A single thick line & A “cusp", or a dihedral angle zero \\
A dashed line & Two divergent faces \\
No line & A dihedral angle $\pi / 2$ \\
\hline
\end{tabular}

A connected Coxeter diagram all of whose proper subdiagrams are elliptic, and the whole diagram is not elliptic or parabolic, is called a Lannér diagram. These correspond to the bounded hyperbolic simplices. A connected Coxeter diagram all of whose proper subdiagrams are elliptic or connected parabolic, and the whole diagram is neither elliptic nor parabolic, is called a quasiLannér diagram. These correspond to the unbounded hyperbolic simplices of finite volume. Complete lists of Lannér and quasi-Lannér diagrams can be found in [10].

\section{Pyramids}

The following two lemmas are straightforward generalisations of Tumarkin's results.

The first lemma was proven for $p=2$ by Tumarkin [7]. This result was based on Vinberg's general construction of unbounded Coxeter polytopes of finite volume which constructed hyperbolic Coxeter pyramids with $n+2$ faces ([1], Chapter 2, §7). Tumarkin then proved the result again for $p=3$. The following Lemma 3.2 is a generalization of Tumarkin's Lemma 11 from [8].

Lemma 3.1. If a hyperbolic Coxeter $n$-polytope $P$ of finite volume is a pyramid with $n+p$ faces, then it is a pyramid over a product of $p$ simplices.

Proof. Suppose that $P$ is a pyramid over some polytope $P^{\prime}$. Then $P^{\prime}$ is the base of the pyramid above which is the apex $A . P^{\prime}$ is bounded by $k$ vertexes, each of which is connected to $A$ by an edge of $P$. All of the faces of $P$ excluding $P^{\prime}$ meet at $A$, and hence it is the confluence of $n+p-1$ faces. When $p=1$ the polytope is a simplex, which is a pyramid over one simplex (of dimension $n-1$ ). For $p>1$ we see that $n+p-1>n$, and so the Coxeter diagram of a vertex is of order greater than $n$. We see from Proposition 2.1 that this forces $A$ to be an infinitely distant vertex. For a sufficiently small horosphere $h$ centred at $A$, the intersection $h \cap P$ is covered by a reflection group. The fundamental domain of this reflection group is a Euclidean Coxeter polytope, which is of the same combinatorial type as $P$ (c.f. [8]).

The geometry of a horosphere is equivalent to that of a Euclidean subspace of dimension $n-1$ in Hyperbolic $n$-space. The hyperbolic Coxeter polytope $P$ is the fundamental domain of a reflection group, which restricts to a Euclidean reflection group which covers the horosphere. Therefore, $h \cap P$ is a bounded Euclidean Coxeter $n-1$ polytope with $n+p-1$ faces. The number of faces in the product of $m$ Euclidean simplices of dimension $l$ is $l+m$, and we solve the following equation.

$$
n+p-1=(n-1)+m \text {. }
$$

Therefore $P^{\prime}$ is equivalent to the product of $p$ simplices. 
The proof of this lemma is like that of Lemma 4 in [7].

Lemma 3.2. Let $P$ be a hyperbolic Coxeter pyramid over a product of $p$ simplices for $p>1$ and $\Sigma$ be a Coxeter diagram of $P$. Then $\Sigma$ satisfies the following three conditions:

1) $\Sigma$ is a union of $p$ quasi-Lannér diagrams $L_{i}$. The intersection of the $L_{i}$ is a unique node $v . L_{i} \backslash v$ and $L_{j} \backslash v$ for $i \neq j$ are not adjacent;

2) Each diagram $L_{i} \backslash v$ is parabolic. Any other subdiagram of $L_{i}$ is elliptic;

3) For any $p$ vertices $\left\{v_{1}, v_{2}, \cdots, v_{n}\right\} \in \Sigma$ such that $v_{i} \in L_{i} \backslash v$ a diagram $\Sigma \backslash\left\{v_{1}, v_{2}, \cdots, v_{n}\right\}$ is either elliptic or connected parabolic.

Any Coxeter diagram satisfying these conditions determines a hyperbolic Coxeter pyramid over a product of $p$ simplices.

Proof. Let $A$ be the apex of the pyramid $P$ over a product of $p$ simplices and $v$ the node of $\Sigma$ corresponding to the face opposite $A$. By Proposition 2.1 as $A$ is an infinitely distant vertex the Coxeter diagram $\Sigma \backslash v$ is parabolic of rank $n-1$. The number of faces in the product of $m$ simplices of dimension $l$ is $l+m$, so the order of the Coxeter diagram is $n+p-1$. For $p>1$ the Coxeter diagram is parabolic and has $p$ connected components which will be denoted $S_{i}, i \in\{1, \cdots, p\}$, all of which are by definition not adjacent. Note that all the subdiagrams of a connected parabolic Coxeter diagram are elliptic.

$\Sigma$ is the Coxeter diagram of a convex polytope of finite volume, and is therefore connected. Hence all of the connected components $S_{i}$ of $\Sigma \backslash v$ are connected to $v$ by an edge, and $\Sigma$ is the union of all of the $L_{i}=S_{i} \cup v$, intersecting in the common node $v$. All other proper subdiagrams of $L_{i}$ determine a face of $P$, and so are elliptic or parabolic. The smallest parabolic diagram is of order two, so the maximum order of a proper subdiagram of an $L_{i}$ is $n+p-1$ and hence for $p>1$ it must be elliptic. We see that, by definition, each of the $L_{i}$ are quasiLannér.

Any vertex of $P$ except $A$ corresponds to a subdiagram $\Sigma \backslash\left\{v_{1}, v_{2}, \cdots, v_{k}\right\}$ such that none of the vertices $v_{i}$ coincide with $v$. If $k>p$ then the order of the resulting diagram is less than $n$, and by Proposition 2.1 it determines a face of codimension $k-p>0$, i.e. it does not determine a vertex. If $k<p$ then the order of the diagram is greater than $n$ and the diagram must be parabolic, and at least one $L_{i}$ remains without any vertices removed. This is a connected component of a parabolic diagram and is therefore parabolic, but it contains a parabolic diagram as a proper subdiagram. Hence $k=p$ and at least one $v_{i}$ must be removed from each $L_{i}$.

Suppose that a Coxeter diagram $\Sigma$ of order $n+p$ satisfies the three conditions of the lemma. Then $\operatorname{det} \Sigma=0$ by Lemma 5.1 in [1]. By an argument identi- cal to that in part 2 of the proof of Lemma 4 in [7] the Coxeter diagram $\Sigma$ determines a Coxeter polytope $P$ in $H^{n}$.

The polytope $P$ is clearly a pyramid over the face $v$. Then by Lemma 3.1 it is a pyramid over a product of $P$ simplices.

These Lemmas provide a precise description of the combinatorial structure of the Coxeter diagram of a hyperbolic Coxeter pyramid. Recall that the hyperbolic pyramids with $n+2$ and $n+3$ faces have been classified by Tumarkin ([7] and [8] respectively) and therefore we need only concern ourselves with $p>3$. We now make use of the above results to find the remaining hyperbolic Coxeter pyramids.

Lemma 3.3. Let $P \subset H^{n}$ be a hyperbolic pyramid with $n+p$ faces, then $p \leq 4$.

Proof. Let $\Sigma$ be the Coxeter diagram of $P$. Choose $v_{i} \in \Sigma, i \in\{1, \cdots, p\}$, which separates $\Sigma$ such that the connected component containing $v$ consists of $v$ and at least one vertex from each of the quasi-Lannér diagrams $L_{i}$. The degree of $v$ in the diagram $\Sigma \backslash v_{i}$ is not less than $p$, and by Lemma 3.2 part (3) the diagram is either elliptic or parabolic. By inspection of the elliptic and parabolic Coxeter diagrams the maximum degree of a vertex is equal to four, which is realised uniquely in the parabolic graph $\tilde{D}_{4}$.

Note that the placement of the parabolic graph $\tilde{D}_{4}$ constrains the labelling of the edges connecting the vertex $v$ to the rest of the graph such that they must all be of weight 3.

Corollary 3.4. Let $P \subset H^{n}$ be a hyperbolic pyramid with $n+4$ faces, then $n=5$.

Proof. Let $\Sigma$ be the Coxeter diagram of $P$. Then $\Sigma$ contains a particular $\tilde{D}_{4}$ as a subgraph, and the vertex of degree four is the base of the pyramid. For $P$ to have finite volume, it is necessary that any parabolic subgraph of $\Sigma$ must be a component of a parabolic graph of rank $n-1$ ([1], Proposition 4.2). Therefore $n-1 \geq 4$.

Assume that $P$ has finite volume, and that $n>5$. Then $\tilde{D}_{4} \subset \Sigma$ is a connected component of $\Gamma^{\prime} \subset \Sigma$, a parabolic graph of rank $n-1$, and the graph $\Gamma=\Gamma^{\prime} \backslash \tilde{D}_{4}$ contains a parabolic graph of rank $n-5$. Therefore the connected components of $\Gamma$ are all parabolic subdiagrams of the quasi-Lannér diagrams $L_{i}$. However, by Lemma 3.2 part 2, each of the $L_{i}$ contain only one parabolic subdiagram, namely $L_{i} \backslash v$, so $\Gamma$ is elliptic. Hence $n=5$.

Proposition 3.5. A hyperbolic pyramid $P$ with $n+4$ faces has a Coxeter diagram which is among those given in Figure 2.

Proof. By Corollary 3.4, hyperbolic pyramids with $n+4$ faces exist in $H^{5}$ only. Therefore we have nine vertices, distributed between four quasi-Lannér diagrams which share a common vertex $v$. The smallest quasi- 
Lannér diagram is a family, each member of which is of rank 2 and has three vertices. Hence each of the four quasi-Lannér diagrams must be from this family, the members of which are shown in Figure 1.

We know that every edge connecting $v$ to another vertex has weight 3 . Therefore the common vertex between all four quasi-Lannér diagrams must be the filled vertex in Figure 1 and the two labels $k$ and $l$ must be either 2 or 3 . We can see that there are only two quasiLannér diagrams with this restriction.

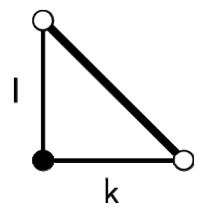

Figure 1. The Coxeter diagrams of the quasi-Lannér diagrams of rank 2 which have the following restrictions: $2 \leq k, l \leq \infty, \frac{1}{k}+\frac{1}{l}<1$.

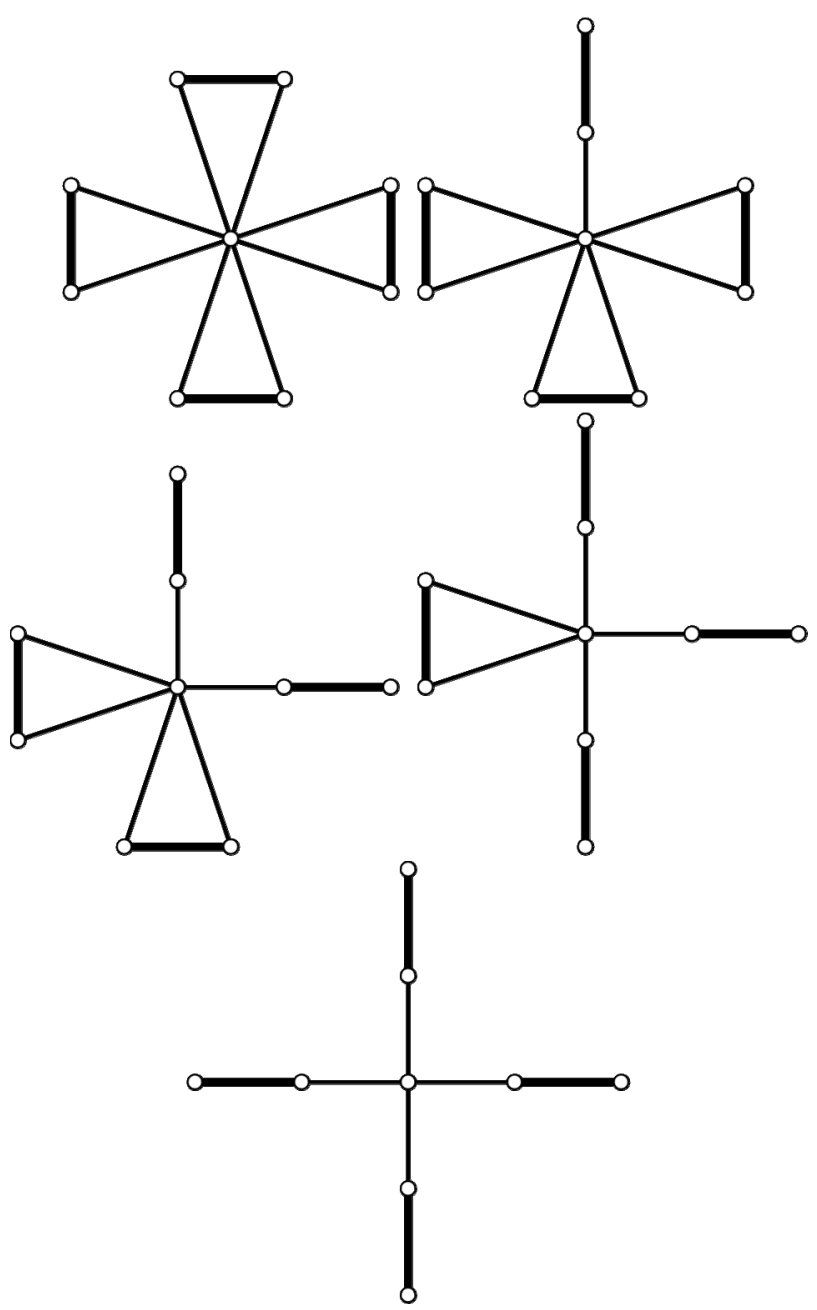

Figure 2. Coxeter diagrams of hyperbolic pyramids with 9 faces in $\boldsymbol{H}^{5}$.
There are five ways to assemble these into a complete Coxeter diagram of a hyperbolic pyramid, and those are presented in Figure 2.

All together, we have proven the following.

Theorem 3.6. Let $P$ be a Coxeter polytope in $H^{n}$ with Coxeter diagram $\Sigma$ of order $n+p$ for $p>1$. The combinatorial type of $P$ is a hyperbolic pyramid over a product of $p$ simplices if and only if it is one of the following:

1) $p=2:$ among the list in Theorem 2 of [7];

2) $p=3$ : among the list in $\S 4$ of [8];

3) $p=4:$ when $\Sigma$ corresponds to a diagram in Figure 2, and this list is complete.

Remark 3.7. The two diagrams in Figure 2 with rotational symmetry of order four were among the root systems listed in Table 5.1 of [11].

\section{Acknowledgements}

The author would like to thank Pavel Tumarkin for his helpful comments on an earlier version of this article, and the anonymous reviewer for his comprehensive assessment of the manuscript.

\section{REFERENCES}

[1] È. B. Vinberg, "Hyperbolic Groups of Reflections," Uspekhi Matematicheskikh Nauk, Vol. 40, 1985, pp. 29-66.

[2] F. Lannér, "On Complexes with Transitive Groups of Automorphisms," Communications du Séminaire Mathématique de l'Université de Lund, Vol. 11, 1950, p. 71.

[3] N. W. Johnson, R. Kellerhals, J. G. Ratcliffe and S. T. Tschantz, "Commensurability Classes of Hyperbolic Coxeter Groups," Linear Algebra and Its Applications, Vol. 345, No. 1, 2002, pp. 119-147. doi:10.1016/S0024-3795(01)00477-3

[4] N. W. Johnson, R. Kellerhals, J. G. Ratcliffe and S. T. Tschantz, "The Size of a Hyperbolic Coxeter Simplex," Transformation Groups, Vol. 4, No. 4, 1999, pp. 329-353. doi:10.1007/BF01238563

[5] M. Chein, "Recherce des Graphes des Matrices de Coxeter Hyperboliques d'Ordre $\leq 10$," Revue Française Informatique Recherche Opérationnelle, Vol. 3, No. 2, 1969, pp. 3-16.

[6] A. A. Felikson, "Coxeter Decomposition of Hyperbolic Simplexes," Sbornik: Mathematics, Vol. 193, No. 12, 2002, pp. 11-12. doi:10.1070/SM2002v193n12ABEH000702

[7] P. V. Tumarkin, "Hyperbolic Coxeter Polytopes in $H^{m}$ with $\mathrm{n}+2$ Hyperfacets," Mathematical Notes, Vol. 75, No. 5-6, 2004, pp. 848-854. doi:10.1023/B:MATN.0000030993.74338.dd

[8] P. V. Tumarkin, "Hyperbolic n-Dimensional Coxeter Polytopes with $\mathrm{n}+3$ Facets," Transactions of the Moscow Mathematical Society, Vol. 58, No. 4, 2004, pp. 235-250.

[9] G. Ziegler, "Lectures on Polytopes," Springer-Verlag, New York, 1995. 
[10] È. B. Vinberg, "Geometry II," Springer-Verlag, Berlin, 1993.

[11] A. A. Felikson and P. V. Tumarkin, "Hyperbolic Subal- gebras of Hyperbolic Kac-Moody Algebras," Transformation Groups, Vol. 17, No. 1, 2012, pp. 87-122.

doi:10.1007/s00031-011-9169-y 